\title{
Cancer immunotherapies targeting the PD-1 signaling pathway
}

Yoshiko Iwai ${ }^{1+}$, Junzo Hamanishi ${ }^{3+}$, Kenji Chamoto ${ }^{2+}$ and Tasuku Honjo ${ }^{2^{*}}$

\begin{abstract}
Immunotherapy has recently emerged as the fourth pillar of cancer treatment, joining surgery, radiation, and chemotherapy. While early immunotherapies focused on accelerating T-cell activity, current immune-checkpoint inhibitors take the brakes off the anti-tumor immune responses. Successful clinical trials with PD-1 monoclonal antibodies and other immune-checkpoint inhibitors have opened new avenues in cancer immunology. However, the failure of a large subset of cancer patients to respond to these new immunotherapies has led to intensified research on combination therapies and predictive biomarkers. Here we summarize the development of PD-1blockade immunotherapy and current issues in its clinical use.
\end{abstract}

Keywords: PD-1, PD-L1, Cancer immunotherapy, Immune checkpoint

\section{Background}

Cancer immunotherapy, although controversial for many years, reached a turning point in 2014. Antibodies that specifically block PD-1 were approved for melanoma in 2014 and for non-small-cell lung cancer (NSCLC) in 2015 in the United States, European Union, and Japan. The success of clinical trials with novel drugs targeting immune-checkpoint molecules such as PD-1 led to a paradigm shift in cancer treatment. Since a PD-1 blockade targets lymphocytes rather than cancer cells, it has a long-term therapeutic effect that persists even when cancers cause mutations. Furthermore, the PD-1 blockade is effective against many types of tumors because it enhances the anti-tumor activity of cytotoxic $\mathrm{T}$ lymphocytes (CTLs), which recognize various tumor-specific antigens. Several companies are currently conducting phase 3 trials for different tumor types, including renalcell cancer (RCC), bladder cancer, head and neck cancer, ovarian cancer, and brain cancer. Although PD-1 blockade has dramatically improved the response rate for several cancers, three questions remain to be answered: 1) Why do some patients not respond to PD-1 blockade? 2) What is the best combination therapy using PD-1

\footnotetext{
* Correspondence: honjo@mfour.med.kyoto-u.ac.jp

${ }^{\dagger}$ Equal contributors

2Department of Immunology and Genomic Medicine, Graduate School of Medicine, Kyoto University, Yoshida Konoe-cho, Sakyo-ku, Kyoto 606-8501, Japan

Full list of author information is available at the end of the article
}

blockade? 3) What predictive biomarkers can be used to distinguish responsive and unresponsive patients? Here we review the development of immunotherapy targeting the PD-1/PD-L1 signaling pathway and discuss the issues that still need to be resolved in clinical studies.

\section{History of cancer immunotherapy}

The concept of cancer immunotherapy goes back to the late nineteenth century. In 1891, a young New York surgeon named William Coley began intra-tumoral injections of bacterial products and observed tumor shrinkage in patients with sarcoma [1]. Almost a century later, the role of dendritic cells and their receptors in sensing microorganisms in the innate immune system was discovered [2,3]. The molecular identification of cancer antigens created new approaches for effective immunotherapies [4]. In addition, the importance of IFN- $\gamma$ and adaptive immunity in cancer immunosurveillance was demonstrated in preclinical tumor models using IFN- $\gamma \mathrm{R}^{-/-}$and $\mathrm{RAG}^{-/-}$mice [5]. These findings stimulated research into strategies to induce anti-tumor responses and led to immunotherapies such as cytokine therapy, peptide vaccine, dendritic-cell vaccine, and adoptive T-cell therapy. Most of these therapies were unsuccessful, and one primary reason was a lack of understanding of the existence and importance of immune checkpoints [6]. 


\section{Immune checkpoints}

T-cell activating (accelerator) and inhibitory (brake) receptors regulate the balance between immune response and immune tolerance. The activation of naïve $\mathrm{T}$ cells requires both antigen presentation (signal 1) and a second signal sent through costimulatory receptors such as CD28 (signal 2) (Fig. 1) [7]. When ligated by B7 molecules such as CD80 (B7-1) or CD86 (B7-2), CD28 coreceptors on $\mathrm{T}$ cells deliver a positive costimulatory signal, whereas CTLA-4 coreceptors deliver a negative coinhibitory signal. PD-1, like CTLA-4, belongs to the CD28 family and delivers a negative signal when it interacts with its ligands, PD-L1 (B7-H1 or CD274) and PDL2 (B7-DC or CD273), which belong to the B7 family (Fig. 1) [8-10].

$\mathrm{T}$ cells have immune checkpoints such as PD-1 and CTLA-4 to reduce autoimmune responses against selftissues by overly exuberant immune responses to infection. While most cancer immunotherapies accelerate Tcell activity, immune-checkpoint inhibitors release the immune system's brakes to unleash anti-tumor immune responses.

\section{Immunoinhibitory mechanism by PD-1}

PD-1 was discovered in 1992 (Fig. 2). Ishida et al. isolated the gene that encodes PD-1 by cDNA subtraction in apoptosis-induced murine T-cell lines. PD-1 is mainly expressed on activated $\mathrm{CD} 4^{+} \mathrm{T}$ cells and $\mathrm{CD} 8^{+} \mathrm{T}$ cells as well as on B cells in the periphery [11-13]. The activation-induced expression of PD-1 suggests that PD1 regulates late-phase immune responses (effector phase, memory response, chronic infection, etc.) in the peripheral tissues, rather than the early induction phase in the lymphoid organs.

PD-1's extracellular region consists of a single IgV-like domain, and its cytoplasmic region contains an immunoreceptor tyrosine-based inhibitory motif (ITIM) and an immunoreceptor tyrosine-based switch motif (ITSM). Upon ligation with its physiological ligand (PDL1 or PD-L2), PD-1 suppresses T-cell activation by recruiting SHP-2, which dephosphorylates and inactivates Zap 70, a major integrator of T-cell receptor (TCR)-mediated signaling $[14,15]$. As a result, PD-1 inhibits the T-cell proliferation and effector functions such as IFN- $\gamma$ production and cytotoxic activity.

The promoter region of the $P d c d 1$ gene has two transcription-factor binding sites that are critical in regulating PD-1 expression. In naïve $T$ cells, TCRmediated calcium influx initiates $P d c d 1$ transcription by activating NFATc1, which binds to the 5 '-promoter region of the $P d c d 1$ gene (at position -1160 relative to the transcription start site) [16]. On the other hand, in chronically activated ("exhausted") $\mathrm{T}$ cells, interferon- $\alpha$ (IFN- $\alpha$ ) causes prolonged $P d c d 1$ transcription by the binding of the transcription factor IRF9 to the $P d c d 1$ promoter (at position -1040 relative to the transcription start site) [17]. In addition, the $P d c d 1$ promoter region (located 500-1500 base pairs upstream of the initiation codon) is demethylated during chronic infection, causing high PD-1 expression in exhausted $\mathrm{CD}^{+} \mathrm{T}$ cells [18]. While exhausted $\mathrm{CD}^{+} \mathrm{T}$ cells express high eomesodermin (EOMES), which is regulated by transcription factor FoxO1, FoxO1 also binds the Pdcd1 promoter and enhances PD-1 expression [19].

\section{PD-1 deficiency and autoimmunity}

PD-1's immunoinhibitory function was elucidated by characterizing the autoimmune phenotype of PD-1-deficient mice, in which PD-1 deficiency leads to a loss of peripheral tolerance and the subsequent development of autoimmunity (Fig. 2) [20, 21]. PD-1-deficient mice develop different autoimmune diseases depending on their

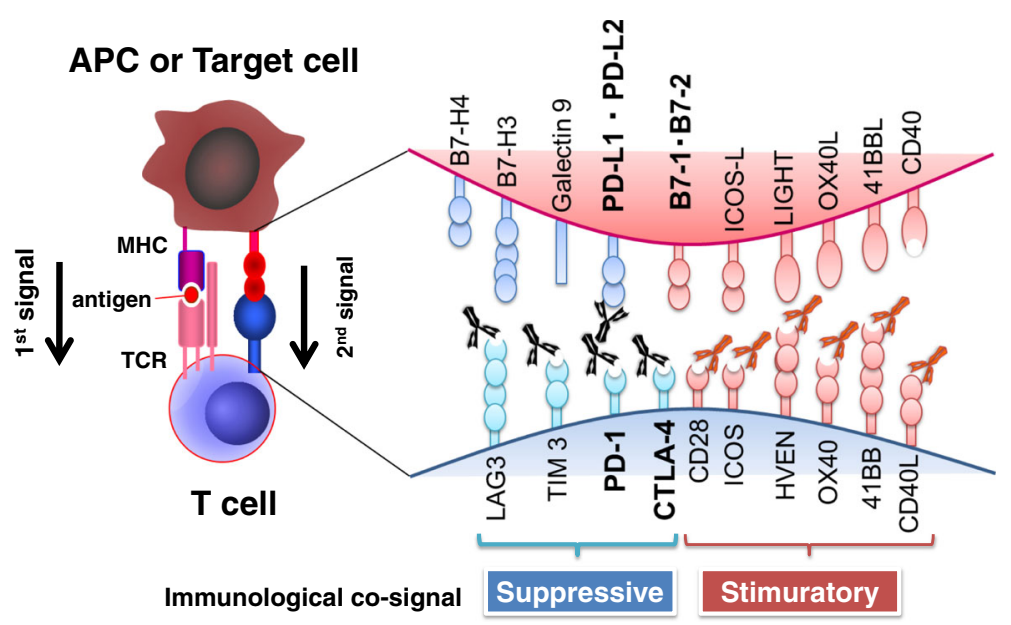

Fig. 1 Costimulatory molecules that positively or negatively regulate immune responses 


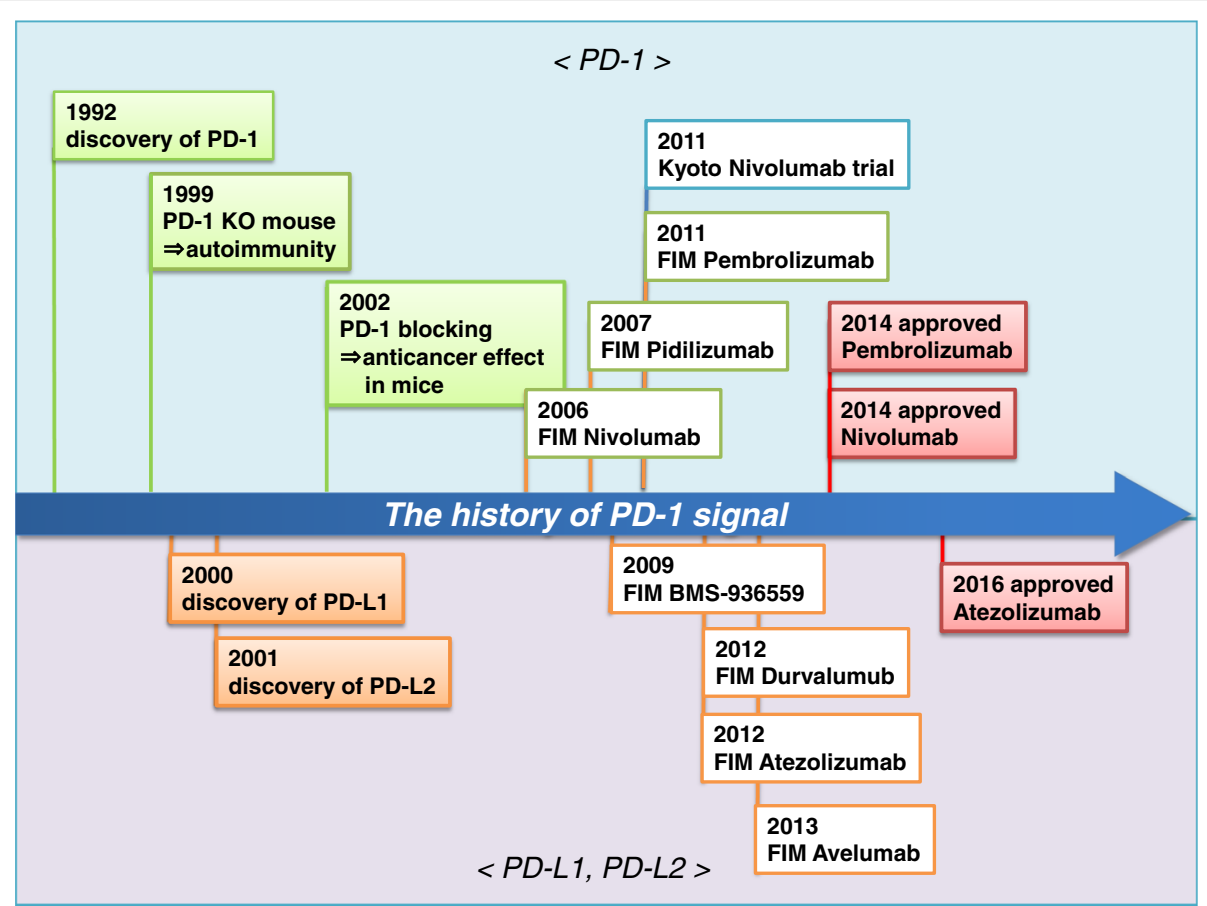

Fig. 2 History of PD-1 research. Abbreviations: FIM, first in man; approved, FDA-approved; NCT, "National Clinical Trial" registry number in ClinicalTrials.gov in the United States; FIM Pembrolizumab (P07990/MK-3475-001/KEYNOTE-001), NCT01295827; FIM Pidilizumab (CT-011), NCT00532259; FIM BMS-936559 (MDX-1105), NCT00729664; FIM Atezolizumab, NCT01693562; FIM Durvalumab (MEDI4736), NCT01693562; FIM Avelumab, NCT01772004

genetic background: C57BL/6-Pdcd1 $1^{-/-}$mice develop lupus-like arthritis and glomerulonephritis with IgG3 and C3 deposits [20]. BALB/c-Pdcd1 ${ }^{-/-}$mice develop fetal dilated cardiomyopathy with a concomitant production of autoantibodies against cardiac troponin I [21, 22]. NOD-Pdcd $1^{-/-}$mice develop type I diabetes with extensive destruction of the islets [23]. Furthermore, PD-1-deficient mice crossed with $\mathrm{H}-2 \mathrm{Ld}$-specific $2 \mathrm{C}$ TCR transgenic mice on the $\mathrm{H}-2 \mathrm{~b} / \mathrm{d}$ background develop a chronic and systemic graft-versus-host-like disease [20]. These findings indicate that PD-1 negatively regulates immune responses and is essential for maintaining peripheral tolerance.

\section{Distinct physiological functions of PD-1 and CTLA-4}

Although PD-1 and CTLA-4 are both induced on activated $\mathrm{T}$ cells, they are expressed at different stages of the immune response. CTLA-4 is closely related to CD28, but binds CD80 and CD86 with a much higher affinity than does CD28 [24]. CTLA-4 is constitutively expressed on regulatory $\mathrm{T}$ (Treg) cells, and transiently expressed on activated $\mathrm{T}$ cells at the early induction phase after antigen stimulation [25]. In contrast, PD-1 is expressed on activated $\mathrm{T}$ cells at the late effector phase, and high and persistent PD-1 expression has been observed on exhausted $\mathrm{CD}^{+} \mathrm{T}$ cells during chronic viral infection $[26,27]$. CTLA-4 is continuously internalized by interactions with the adaptor complex AP2 and is almost undetectable on the cell surface during T-cell activation; in contrast, PD-1 lacks an AP2-binding motif, which may allow its sustained expression on the surface of activated $\mathrm{T}$ cells [28].

Although both PD-1 and CTLA-4 are immune checkpoints, they regulate different phases of the immune response. CTLA-4 blocks early T-cell activation in the lymphoid organs, whereas PD-1 inhibits effector T-cell activity at later-stage immune responses in peripheral tissues and in the tumor microenvironment. PD-1 and CTLA-4 also have distinct inhibitory mechanisms. CTLA-4 completely blocks costimulation by CD28 through its stronger affinity for B7 molecules, whereas PD-1's inhibitory function depends mostly on its recruitment of SHP-2 [29-32]. These differences in expression and inhibitory mechanisms are probably responsible for the different autoimmune phenotypes of PD- 1 and CTLA-4 deficiency. CTLA-4deficient mice develop devastating autoimmune diseases and massive and systemic lymphoproliferation, and die within 5 weeks of birth [33]. In contrast, PD1 -deficient mice remain relatively healthy into later stages of life, eventually developing relatively mild, organ-specific autoimmune symptoms depending on their genetic background [20,21]. Consistent with the phenotypes of PD-1-knockout and CTLA-4-knockout 
mice, PD-1 inhibitors are less toxic than CTLA-4 inhibitors [34, 35].

\section{Identification of PD-1 ligands}

PD-L1 and PD-L2 were identified as PD-1 ligands in 2000 and 2001, respectively (Fig. 2) [9, 10]. PD-L1 and PD-L2 are type I transmembrane proteins with IgV- and IgC-like domains in the extracellular region. PD-L1 is broadly expressed in both lymphoid and non-lymphoid tissues. PD-L1 is upregulated upon activation on hematopoietic cells, especially on antigen-presenting cells (APCs) such as dendritic cells, macrophages/monocytes, and B cells $[36,37]$. PD-L1 is also expressed on activated $\mathrm{T}$ cells. Importantly, PD-L1 is expressed on non-lymphoid cells, including parenchymal cells and vascular endothelial cells in the peripheral tissues, and is upregulated by IFN- $\gamma$ and other inflammatory cytokines secreted by activated $\mathrm{T}$ cells $[23,26,38]$. The expression of PD-L1 in peripheral tissues rather than on professional APCs is crucial for preventing autoimmune damage to tissues [39]. Interestingly, PD-L1 is expressed in various tumor cells and virus-infected cells. The expression of PD-L1 on target cells allows PD-1 to directly inhibit T-cell effector functions against the target cell. Unlike PD-L1, which is expressed in many different tissues, PD-L2 is expressed only on APCs such as dendritic cells and macrophages [37].

\section{Regulation of tumor immunity by PD-1}

The PD-1/PD-L1 signaling pathway is crucial in dampening immunosurveillance for tumors. Tumors can escape host immune surveillance by expressing PD-L1, which negatively regulates immune responses by interacting with PD-1 on T cells (Fig. 1) [40]. Indeed, data from clinical samples indicate that the high expression of PD-1 ligands on tumors is correlated with a poor prognosis $[41,42]$.

The first evidence of the PD-1/PD-L1 pathway's involvement in tumor immunity was found in animal models [40]. PD-L1 overexpression on P815 mastocytomas was shown to inhibit the cytolytic activity of $\mathrm{CD} 8^{+}$ $\mathrm{T}$ cells by engaging PD-1 in vitro, and to markedly enhance tumorigenesis and tumor invasiveness in vivo. Anti-PD-L1 treatment inhibited the growth of PD-L1expressing P815 tumor cells, and of J558L myeloma cells, which endogenously express PD-L1. Importantly, no tumors developed in $P d c d 1^{-/-}$mice after their inoculation with J558L cells. These results revealed the effectiveness of the PD-1/PD-L1 blockade for tumor therapy.

Although a CTLA-4 blockade enhances immune responses against immunogenic tumors such as lymphoma in animal models, it is not effective as a single agent against poorly immunogenic tumors such as B16 melanoma [43-45]. However, even as a single agent, a PD-1 blockade was found to be therapeutic against B16 melanoma in a liver metastasis model [46]. These results suggested that PD-1 blockade can be successfully applied to metastatic tumors, and that it has a stronger therapeutic potential than does CTLA-4 blockade.

\section{Clinical application of the PD-1/PD-L1 blockade}

Several clinical studies have reported that PD-L1 overexpression is related to a poor prognosis for several types of tumors, including renal-cell carcinoma, bladder cancer, esophageal cancer, pancreatic cancer, gastric cancer, hepatocellular carcinoma, and ovarian cancer [41, 4753]. In ovarian cancer, PD-L1 expression is negatively correlated with the number of intra-epithelial infiltrating $\mathrm{CD}^{+} \mathrm{T}$ cells, suggesting that the PD-L1 expression on tumor cells prevents $\mathrm{CD}^{+} \mathrm{T}$ cells from infiltrating tumor sites [50]. These studies indicated that blocking PD-1 signaling might improve clinical outcomes for patients with these malignancies. In 2006, a proof-ofconcept clinical study using a PD-1 signal inhibitor against treatment-resistant solid tumors was initiated in the United States (Fig. 2) [54].

A fully humanized monoclonal antibody $(\mathrm{mAb})$ against PD-1 (nivolumab; also known as ONO4538, MDX-1106, or BMS-936558) was first developed using genetically modified mice carrying loci encoding human immunoglobulins. The IgG4 isotype of nivolumab minimizes complement activity or antibody-dependent cellmediated cytotoxicity (ADCC) [54]. This antibody carries a serine-to-proline substitution at position 228 to minimize the effect of ADCC against activated T cells. Clinical trials of nivolumab began in 2006 in the United States and in 2009 in Japan (Fig. 2). The phase 1 study of nivolumab showed cumulative response rates of $18 \%$ for NSCLC, $28 \%$ for melanoma, and $27 \%$ for renal carcinoma. Grade 3 or 4 drug-related adverse events occurred in $14 \%$ of the patients [35]. Notably, nivolumab has demonstrated durable clinical activity as a single agent, with far fewer side effects than are seen with ipilimumab, a mAb against CTLA-4 [34, 35]. A clinical trial using anti-PDL1 mAbs (BMS-936559 or MDX-1105) showed relatively low response rates compared to an anti-PD-1 mAb [55].

The PD-1 blockade approach has unique features compared to standard therapies. Conventional chemotherapies usually target a particular molecule in the tumor cells. The tumor cells can escape the therapy with mutations of the target molecules, leading to rapid regression. However, a PD-1 blockade is applicable to a wide range of cancers and provides a response over a longer period because it activates an anti-tumor immune system that can target mutated proteins [56]. In addition, PD-1 blockade has a significantly lower rate of high-grade toxicities than other immunotherapies or 
standard therapies, because the anti-tumor immunity preferentially recognizes tumor-derived antigens, not self-antigens. In a phase 3 study comparing nivolumab to the plant alkaloid chemotherapy drug docetaxel in 272 patients with advanced squamous-cell NSCLC, the response rate was $20 \%$ with nivolumab versus $9 \%$ with docetaxel [57]. The overall survival rate at 1 year was $42 \%$ with nivolumab versus $24 \%$ with docetaxel. The frequency of grade 3 or 4 treatment-related adverse events was much lower in the nivolumab group (7\%) than in the docetaxel group (55\%).

To date, at least 500 clinical studies with PD-1 signal inhibitors have been conducted with nine types of antibodies from eight pharmaceutical companies (Table 1 and Fig. 2) on at least 20 types of solid and hematological malignant tumors (Table 2) [58]. The total number of subjects worldwide is more than 20,000, according to a clinical trials database managed by the U.S. National Institutes of Health (https://clinicaltrials.gov/ [CTG]). The U.S. Food and Drug Administration (FDA) approved nivolumab for patients with unresectable or metastatic melanoma in 2014, for NSCLC in 2015, and for classical Hodgkin's lymphoma and RCC in 2016. The FDA also approved pembrolizumab for melanoma in 2014 and for NSCLC in 2015. Atezolizumab, an antiPD-L1 antibody, was approved for unresectable bladder cancer and for NSCLC in 2016.

Regarding clinical trials for ovarian cancer, we first conducted the principal investigator-initiated two-cohort
( 1 or $3 \mathrm{mg} / \mathrm{kg}, n=10$ each) phase 2 clinical trial of nivolumab in 20 patients with platinum-resistant recurrent ovarian cancer at Kyoto University Hospital in 2011 (Fig. 2) (UMIN000005714) [59, 60]. The objective response rate at $3 \mathrm{mg} / \mathrm{kg}$ was $20 \%$; this included two cases of complete response (CR). For all 20 patients, the response rate was $15 \%$ and the durable CR (DCR) was $45 \%$. The median progression-free survival (PFS) and overall survival (OS) were 3.5 and 20.0 months, respectively [60]. In our ongoing follow-up study of this trial, a durable anti-tumor response with nivolumab has been observed in two patients with a complete response for over 1 year. After completing the 1 -year nivolumab treatment, these two patients survived without any antitumor treatment for over 2 years [61, 62]. Based on this positive result, we are conducting a large randomized phase 2 trial with nivolumab versus standard 2nd-line chemotherapy (NINJA study, JapicCTI-153004). So far, at least 30 clinical trials have been completed or are underway for ovarian cancers using the monotherapeutic anti-PD-1 antibody pembrolizumab (response rate [RR] $10 \%, n=104)$ [63], the anti-PD-L1 antibody avelumab (RR $10 \%, n=104)$ [64], or combinations of these agents with conventional cancer therapies (CTG).

\section{Combination therapy with blockade of PD-1/PD- L1 signal and new co-signals}

Patients who respond poorly or are unresponsive to immunotherapies appear to lack preexisting anti-tumor T-

Table 1 PD-1 signal inhibitors (anti-PD-1 and anti-PD-L1 antibodies) in clinical trials

\begin{tabular}{|c|c|c|c|c|}
\hline Target & Agent & IgG class & Company & Approved \\
\hline \multirow[t]{7}{*}{$\overline{\mathrm{PD}-1}$} & nivolumab (Opdivo ${ }^{\oplus}$, BMS-936558, MDX1106) & Human IgG4 & Bristol-Meyers Squibb/Ono & 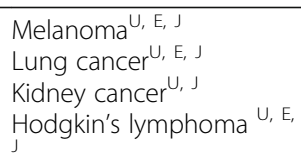 \\
\hline & & & & $\begin{array}{l}\text { Head and neck cancer }{ }^{\cup} \\
\text { Urothelial cancer }{ }^{\cup}\end{array}$ \\
\hline & $\begin{array}{l}\text { pembrolizumab (Keytruda®MK-3475, } \\
\text { lambrolizumab) }\end{array}$ & Humanized lgG4 & Merck & $\begin{array}{l}\text { Melanoma }{ }^{U, E}, J \\
\text { Lung cancer }^{U}, \mathrm{E}, J \\
\text { Head and neck cancer }\end{array}$ \\
\hline & pidilizumab (CT-011) & Humanized lgG1k & Cure Tech & \\
\hline & AMP-224 & $\begin{array}{l}\text { PD-L2 IgG2a fusion } \\
\text { protein }\end{array}$ & $\begin{array}{l}\text { Amplimmune/GlaxoSmith } \\
\text { Klein }\end{array}$ & \\
\hline & AMP-514 (MEDI0680) & PD-L2 fusion protein & $\begin{array}{l}\text { Amplimmune/GlaxoSmith } \\
\text { Klein }\end{array}$ & \\
\hline & PDR001 & Humanized lgG4 & Novartis Pharmaceuticals & \\
\hline \multirow[t]{4}{*}{ PD-L1 } & BMS-936559 (MDX1105) & Human lgG4 & Bristol-Meyers Squibb & \\
\hline & atezolizumab (Tecentriq ${ }^{\oplus}$, MPDL3280A) & Humanized lgG1k & Roche/Genentech & $\begin{array}{l}\text { Urothelial cancer }{ }^{U} \\
\text { Lung cancer }\end{array}$ \\
\hline & durvalumab (MEDI4736) & Human lgG1k & Medlmmune/AstraZeneca & \\
\hline & avelumab (MSB0010718C) & Human IgG1 & Merck Serono/Pfizer & \\
\hline
\end{tabular}

All antibodies used in clinical trials as of September 1, 2016 were extracted from ClinicalTrials.gov

Abbreviations: U U.S. Food and Drug Administration (FDA) approved; E European Medicines Agency (EMA) approved, $J$ Japanese Pharmaceutical and Medical Devices Agency $(P M D A)$ approved 
Table 2 Clinical effects of monotherapeutic PD-1 signal inhibitors on several types of malignancies

\begin{tabular}{|c|c|c|c|c|}
\hline Target & Agent & Phase & Clinical effect & Reference \\
\hline \multirow[t]{4}{*}{ melanoma } & \multirow[t]{2}{*}{ pembrolizumab } & 2 & 6 MOS 34\% (2 mg/kg) vs. 38\% (10 mg/kg), vs $16 \%$ :docetaxel $(n=540)$ & {$[88]$} \\
\hline & & 3 & 1 year-OS 74\% (2wks) vs. 38\% (3wks), vs 11\% :docetaxel $(n=834)$ & {$[89]$} \\
\hline & \multirow[t]{2}{*}{ nivolumab } & 3 & 1 year-OS $73 \%$ vs $42 \%$ (dacarbazine) $(n=418)$ & {$[89]$} \\
\hline & & 3 & ORR $32 \%$ vs. $11 \%$ (dacarbazine) $(n=405)$ & {$[90]$} \\
\hline \multirow[t]{5}{*}{ non-small cell lung cancer } & pembrolizumab & 1 & ORR 19.4\%, mOS12.5 M (total), ORR 45.2\% ( $n=72$, PD-L1+) $(n=495)$ & {$[91]$} \\
\hline & \multirow[t]{2}{*}{ nivolumab } & 3 & mOS 9.2 M (vs 6.0 M:docetaxel) $(n=272)$ & {$[57]$} \\
\hline & & 3 & mOS12.2 M (vs 9.7 M:docetaxel $(n=582)$ & {$[92]$} \\
\hline & durvalumab & $1 / 2$ & ORR 14\% $(n=149$, total), 23\% (PD-L1+) & {$[72]$} \\
\hline & atezolizumab & 2 & ORR 15\% $(n=144$, total $), 38 \%(n=24$, PD-L1+) & [93] \\
\hline \multirow[t]{2}{*}{ small cell lung cancer } & nivolumab & $1 / 2$ & ORR $18 \%$ ( $n=40$, nivo), $17 \%$ ( $n=46$, combined with chemotherapy) & [94] \\
\hline & pembrolizumab & 1 & ORR $25 \%(n=16)$ & {$[95]$} \\
\hline \multirow[t]{2}{*}{ head and neck cancer } & durvalumab & $1 / 2$ & ORR $12 \%(n=62)$ & {$[96]$} \\
\hline & pembrolizumab & 1 & ORR $24.8 \%(n=117)$ & {$[97]$} \\
\hline renal cell cancer & nivolumab & 3 & $\begin{array}{l}\text { ORR 25\%, mOS } 25.0 \mathrm{M} \text {, (vs. ORR 5\%, mOS 19Ms in everolimus) } \\
(n=821)\end{array}$ & {$[98]$} \\
\hline \multirow[t]{2}{*}{ bladder cancer } & atezolizumab & 1 & ORR 26\% ( $n=310$, total), 43\% (PD-L1+) & [99] \\
\hline & pembrolizumab & 1 & ORR 25\% ( $n=33$, total), 38\% (PD-L1+) & {$[100]$} \\
\hline \multirow[t]{3}{*}{ ovarian cancer } & nivolumab & 2 & ORR 15\% ( $n=20$, total), mOS 20.0 M ORR 20\% $(n=10,3 \mathrm{mg} / \mathrm{kg})$ & {$[60]$} \\
\hline & avelumab & 1 & ORR $10 \%(n=124)$ & {$[101]$} \\
\hline & pembrolizumab & 1 & ORR 11.5\% (PD-L1+) $(n=49)$ & {$[63]$} \\
\hline uterine endometrial cancer & pembrolizumab & 1 & ORR $12.5 \%(\mathrm{PD}-\mathrm{L} 1+)(n=24)$ & [102] \\
\hline uterine cervical cancer & pembrolizumab & 1 & ORR 12.5\% (PD-L1+) $(n=24)$ & [103] \\
\hline uterine sarcoma & nivolumab & 1 & ORR 0\% $(n=12)$ & [104] \\
\hline gastric cancer & pembrolizumab & 1 & ORR $31 \%(n=39)$ & {$[79]$} \\
\hline esophageal cancer & pembrolizumab & 1 & ORR 30\% (PD-L1+) $(n=23)$ & {$[105]$} \\
\hline DNA mismatch repair deficient colon & pembrolizumab & 2 & $\begin{array}{l}\text { ORR } 40 \%(n=10, \text { MMRd colon), vs } 0 \%(n=18) \text { in MMRw, vs } 71 \%(n=7) \text {, } \\
\text { MMR-non-colon [cholangiocarcinoma, endometrial cancer and } \\
\text { pancreatic cancer].) }\end{array}$ & [73]. \\
\hline $\begin{array}{l}\text { DNA mismatch repair deficient } \\
\text { endometrial cancer }\end{array}$ & pembrolizumab & 2 & ir-ORR 67\% $(n=9)$ & {$[106]$} \\
\hline hepatocellular carcinoma & nivolumab & $1 / 2$ & ORR 9\% $(n=91), 6$ month-OS 69\%. & {$[107]$} \\
\hline \multirow[t]{2}{*}{ breast cancer } & atezolizumab & 1 & ORR $12 \%(n=27)$ & {$[108]$} \\
\hline & pembrolizumab & 1 & ORR 19\% $(n=25)(\mathrm{PD}-\mathrm{L} 1+)$ & [109] \\
\hline Merkel cell carcinoma & pembrolizumab & 2 & ORR $56 \%(n=25), 6$ M-PFS $67 \%$ & {$[110]$} \\
\hline thyroid cancer & pembrolizumab & 1 & ORR 9.1\% $(n=22)$, mOS not reached, 1 year-OS 89.9\%. & [1111] \\
\hline \multirow[t]{2}{*}{ Hodgikin lymphoma } & nivolumab & 1 & ORR $87 \%, 24 w k s-P F S ~ 86 \%(n=23)$ & {$[112]$} \\
\hline & pembrolizumab & 1 & ORR $64 \%(n=31), 52 w k s-P F S ~ 46 \%$. & [113] \\
\hline follicular lymphoma & nivolumab & 1 & ORR $40 \%(n=10)$ & {$[114]$} \\
\hline diffuse large B-cell lymphoma & nivolumab & 1 & ORR $36 \%(n=11)$ & [114] \\
\hline mycosisfungoides & nivolumab & 1 & ORR 15\% $(n=13)$ & [114] \\
\hline peripheral T-cell lymphoma & nivolumab & 1 & ORR $40 \%(n=5)$ & [114] \\
\hline
\end{tabular}

Partially modified from reference [58]. Abbreviations: $M$ month, wk week, ORR objective response rate, OS overall survival, $P F S$ progression-free survival, irRC immune-related response criteria, ASCO Annual meeting of the American Society of Clinical Oncology, SGO Annual meeting of the Society of Gynecologic Oncology, Abst Abstract, MMRd DNA mismatch repair deficient, MMRw DNA mismatch repair wild 
cell responses. One possible approach to overcoming this issue is to combine the two immune-checkpoint inhibitors anti-PD-1 and anti-CTLA-4 (Fig. 1). In a phase 1 study on patients with advanced melanoma, concurrent therapy with nivolumab and ipilimumab induced rapid and durable responses, resulting in an unprecedented 2-year survival rate of over $80 \%$; $53 \%$ of the patients had an objective response with more than $80 \%$ tumor reduction [65]. However, grade 3 or 4 therapy-related adverse events occurred in $53 \%$ of the patients. In this double-blind study, 142 patients with metastatic melanoma randomly received ipilimumab combined with nivolumab or placebo once every 3 weeks for four doses. The objective response rate was significantly higher for patients who received the combined ipilimumab and nivolumab regimen (60\%) compared to those treated with ipilimumab monotherapy (11\%). The median PFS was 8.9 months with the combination therapy and 4.7 months with ipilimumab alone [66]. Based on this confirmatory trial, the FDA approved this combined nivolumab and ipilimumab therapy for unresectable or metastatic melanoma in 2015. Combined nivolumab and ipilimumab therapy is now being clinically applied to other cancer types, including RCC [67], NSCLC [68], and ovarian cancer (NCT02498600). However, the frequency of grade 3 or 4 immunerelated adverse events (irAEs) is over 50\%, and this issue remains to be resolved [69].

Several clinical trials are underway for PD-1 inhibitors in combination with other immune-checkpoint inhibitors, immune activators, and chemotherapies (Table 3 and Fig. 1). However, combining immunotherapies with chemotherapies can increase irAEs. For example, compared to PD-L1 mAb (durvalumab) or EGFR inhibitor (osimertinib) monotherapies, combining the two therapies induced a significantly higher risk of interstitial lung disease ( $2 \%$ [ $n=23$ of 1149], 2.8\% [35 of 1207], and 38\% [ $n=13$ of 34], respectively) [70]. At present, at least 20 clinical trials with combined PD-1 inhibitors and focal radiation therapy and more than five trials combining anti-PD-1 mAb with chemoradiation therapy are underway (CTG).

\section{Biomarkers for predicting the efficacy of the PD- 1-blockade cancer immunotherapy}

Potential predictive biomarkers for anti-tumor responses with PD-1 inhibitors can be found among both tumor cell-related factors and host immunological factors. Recent reports identified the frequency of genetic mutations derived from microsatellite instability (MSI) with DNA mismatch repair deficiency (MMRd) in cancer cells as a candidate biomarker [56, 71-73]. Many mutated neo-antigens expressed on the surface of cancer cells are recognized by $\mathrm{T}$ cells and $\mathrm{B}$ cells as foreign antigens, either directly or through the APC system. Cancer cells exposed to IFN- $\gamma$ released from activated T cells express PD-L1, thereby establishing an acquired immune resistance [74]; in this case, PD-1 signal inhibitors are more likely to be effective. Thus, genome-wide mutation analysis (i.e., Mutanome) of cancer cells using next-generation sequencing technology and diversity analysis of the T-cell or B-cell repertoire (i.e., Immunome) have attracted a lot of attention as strategies for identifying predictive biomarkers (Fig. 3) [75, 76]. Based on this concept, researchers have examined candidate biomarkers such as the PD-L1 level on tumor cells and the frequency of tumor-infiltrating lymphocytes [77, 78], the levels of IFN- $\gamma$-related genes in tumor cells $[79,80]$, the frequency of mutations in tumor cells [56, 71-73], and the diversity of TCRs in tumor antigen-specific $\mathrm{T}$ cells $[74,81,82]$. However, these candidates do not always correspond to a high response according to cancer type. For example, clinical trials of PD-1 inhibitors for squamous-cell lung cancer and ovarian cancer showed no correlation between clinical effect and PD-L1 expression on tumor tissues [57, 60, 64]. A recent report by Hugo et al. revealed that high mutational loads and genes related to $T$-cell checkpoints, such as CD8A/B, PD-L1, LAG3, and IFN- $\gamma$, in tumor tissues were not associated with responsiveness in breast cancer patients [83]. Interestingly, the breast cancer susceptibility gene (BRCA) 2 mutation status is associated with responsiveness to PD-1 mAb therapy [83], while no correlation was found between BRCA2 and avelumab's clinical effect on ovarian cancer [64]. It is urgent to validate current candidates and to discover new biomarkers for clinical response to PD-1 signal inhibitors.

\section{Toxicities of PD-1/PD-L1 signal blocking}

IrAEs associated with PD-1 blockade therapy include interstitial pneumonitis, colitis with gastrointestinal perforation, type 1 diabetes, severe skin reactions, immune thrombocytopenia, neutropenia and sepsis after corticosteroid therapy, encephalopathy and neurological sequelae, Guillain-Barré syndrome, myelitis, myasthenia gravis, myocarditis and cardiac insufficiency, acute adrenal insufficiency, and nephritis [84-87]. Based on several previous clinical trials, guidelines and specific care algorithms have been established for the identification, early intervention, and management of irAEs [86, 87]. While irAEs can develop at any time, most of the immune toxicities of nivolumab occur within the first 4 months [86, 87]. The median time to onset of irAEs tends to differ depending on the type of toxicity, and can be roughly classified as early $(<2$ months: skin, gastrointestinal, or hepatic irAEs) or late ( $>2$ months: pulmonary, endocrine, and renal-related irAEs). To treat new types of adverse events and to reduce the frequency 
Table 3 Clinical trials of combination therapies with molecularly targeted drugs

\begin{tabular}{llll}
\hline PD-1/PD-L1mAb & Combination & Tumor & Reference \\
\hline PD-1 mAb (Nivolumab) & LAG3 (BMS-986016) & Solid Tumors & NCT01968109 \\
PD-1 mAb (Nivolumab) & B7-H3 (Enoblituzumab) & Solid Tumors & NCT02817633 \\
PD-1 mAb (Pembrolizumab) & B7-H3 (Enoblituzumab) & Solid Tumors & NCT02475213 \\
PD-1 mAb (Nivolumab) & KIR (Lirilumab) & Solid Tumors & NCT01714739 \\
PD-L1 mAb (MEDI4736) & OX40 (MEDI6383) & Solid Tumors & NC221960 \\
PD-1 mAb (Nivolumab) & 4-1BB (Urelumab) & Solid tumors and B-cell non-Hodgkin lymphoma & NCT02253992 \\
PD-1 mAb (Nivolumab) & ICOS (JTX-2011) & NCT02904226 \\
Pd-1 mAb (PDR001) & GITR (GWN323) & Solid Tumors & NCT02740270 \\
PD-1 mAb (Nivolumab) & CD27 (Varlilumab) & Solid Tumors and Lymphomas & NCT02335918 \\
PD-L1 mAb (Atezolizumab) & CD27 (Varlilumab) & Solid Tumors & NCT02543645 \\
PD-1 mAb (Nivolumab) & GM.CD40L (vaccine for NSCLC) & Solid Tumors & Lung (NSCLC) \\
PD-L1 mAb (Atezolizumab) & VEGF inhibitors (Bevacizumab cediranib) & Ovarian Cancer & NCT02659384 \\
PD-L1 mAb (MEDl4736) & PARP inhibitors (Olaparib) & S tumors \\
PD-L1 mAb (MEDI4736) & Multi-kinase inhibitor (Sunitinib) & Solid tumors \\
PD-1 mAb (Pembrolizumab) with SBRT & Multi-kinase inhibitor (Sunitinib) & TKl refractory mRCC & NCT02484404 \\
PD-L1 mAb (Durvalumab) & EGFR inhibitor (Osimertinib) & Lung (NSCLC) & NCT02484404 \\
\hline
\end{tabular}

${ }^{a}$ Tyrosine kinase inhibitor refractory metastatic recal cell cancer

of immunological toxicities, oncologists should form and collaborate with networks of organ-specific medical doctors, pharmacists, and nurses.

\section{Conclusion}

Basic and translational studies in the 20 years since PD1 's discovery have demonstrated the concept of immune surveillance in mice and humans. The recovery of T-cell anergy by blocking PD-1 signals on $\mathrm{T}$ cells yielded incredible clinical benefits for several types of malignancies. Nevertheless, there is a still a great deal of exploratory research needed to clarify the fundamental mechanism and predictive biomarkers for the efficacy and adverse effects of this therapeutic strategy. To advance the development of PD-1 signal inhibitors in cancer therapy, it is important to continue both

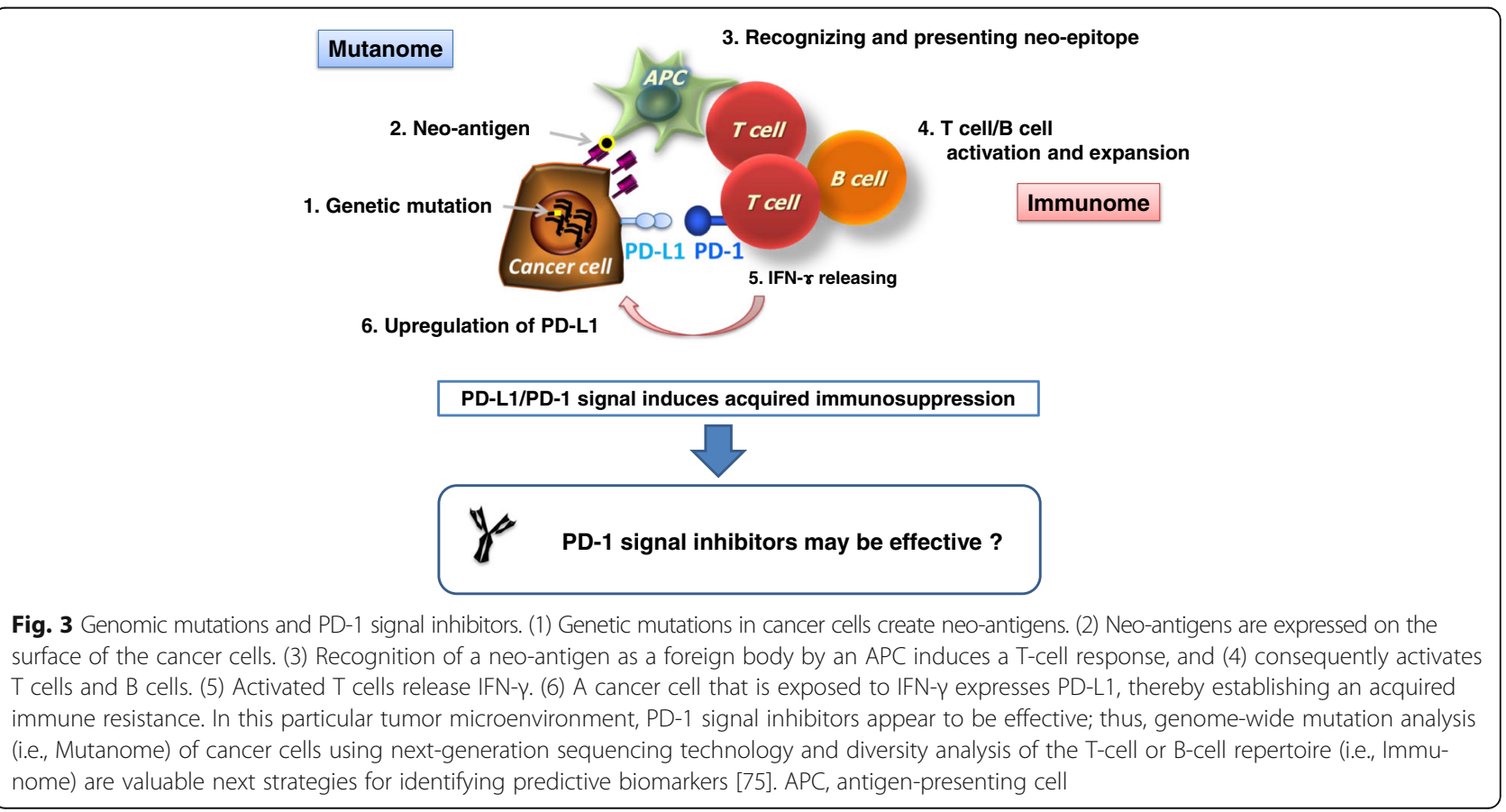


translational and reverse-translational research approaches, including molecular and genomic studies to elucidate the interactions between host and tumor cells.

\section{Acknowledgements}

None.

\section{Funding}

None.

\section{Availability of data and materials}

Not applicable.

\section{Authors' contributions}

$I Y, H J$, and CK are eaqually contributed. All authors read and approved the final manuscript.

\section{Authors' information}

None.

\section{Competing interests}

The authors declare that they have no competing interests.

\section{Consent for publication}

None.

\section{Ethics approval and consent to participate}

Not applicable.

\section{Publisher's Note}

Springer Nature remains neutral with regard to jurisdictional claims in published maps and institutional affiliations.

\section{Author details \\ 'Department of Molecular Biology, School of Medicine, University of Occupational and Environmental Health Japan, Kitakyushu-shi, Fukuoka 807-8555, Japan. ${ }^{2}$ Department of Immunology and Genomic Medicine, Graduate School of Medicine, Kyoto University, Yoshida Konoe-cho, Sakyo-ku, Kyoto 606-8501, Japan. ${ }^{3}$ Department of Gynecology and Obstetrics, Graduate School of Medicine, Kyoto University, 54 Kawahara-cho, Shogoin, Sakyo-ku, Kyoto 606-8507, Japan}

Received: 10 March 2017 Accepted: 20 March 2017

Published online: 04 April 2017

\section{References}

1. Mellman I, Coukos G, Dranoff G. Cancer immunotherapy comes of age. Nature. 2011:480:480-9.

2. Steinman RM, Cohn ZA. Identification of a novel cell type in peripheral lymphoid organs of mice. I. Morphology, quantitation, tissue distribution. $J$ Exp Med. 1973:137:1142-62.

3. Lemaitre B, Nicolas E, Michaut L, Reichhart JM, Hoffmann JA. The dorsoventral regulatory gene cassette spatzle/Toll/cactus controls the potent antifungal response in Drosophila adults. Cell. 1996;86:973-83.

4. van der Bruggen $P$, et al. A gene encoding an antigen recognized by cytolytic T lymphocytes on a human melanoma. Science. 1991;254:1643-7.

5. Schreiber RD, Old LJ Smyth MJ. Cancer immunoediting: integrating immunity's roles in cancer suppression and promotion. Science. 2011;331: 1565-70.

6. Pardoll DM. The blockade of immune checkpoints in cancer immunotherapy. Nat Rev Cancer. 2012;12:252-64.

7. Lenschow DJ, Walunas TL, Bluestone JA. CD28/B7 system of T cell costimulation. Annu Rev Immunol. 1996;14:233-58.

8. Okazaki T, Chikuma S, Iwai Y, Fagarasan S, Honjo T. A rheostat for immune responses: the unique properties of PD-1 and their advantages for clinical application. Nat Immunol. 2013;14:1212-8.

9. Freeman GJ, et al. Engagement of the PD-1 immunoinhibitory receptor by a novel B7 family member leads to negative regulation of lymphocyte activation. J Exp Med. 2000;192:1027-34.
10. Latchman Y, et al. PD-L2 is a second ligand for PD-1 and inhibits T cell activation. Nat Immunol. 2001:2:261-8.

11. Ishida Y, Agata Y, Shibahara K, Honjo T. Induced expression of PD-1, a novel member of the immunoglobulin gene superfamily, upon programmed cell death. EMBO J. 1992;11:3887-95.

12. Agata $Y$, et al. Expression of the PD-1 antigen on the surface of stimulated mouse T and B lymphocytes. Int Immunol. 1996;8:765-72.

13. Iwai Y, et al. Microanatomical localization of PD-1 in human tonsils. Immunol Lett. 2002:83:215-20.

14. Okazaki T, Maeda A, Nishimura H, Kurosaki T, Honjo T. PD-1 immunoreceptor inhibits B cell receptor-mediated signaling by recruiting src homology 2-domain-containing tyrosine phosphatase 2 to phosphotyrosine. Proc Natl Acad Sci U S A. 2001:98:13866-71.

15. Yokosuka T, et al. Programmed cell death 1 forms negative costimulatory microclusters that directly inhibit T cell receptor signaling by recruiting phosphatase SHP2. J Exp Med. 2012;209:1201-17.

16. Oestreich KJ, Yoon H, Ahmed R, Boss JM. NFATc1 regulates PD-1 expression upon T cell activation. J Immunol. 2008;181:4832-9.

17. Terawaki $S$, et al. IFN-alpha directly promotes programmed cell death-1 transcription and limits the duration of T cell-mediated immunity. J Immunol. 2011:186:2772-9.

18. Youngblood $B$, et al. Chronic virus infection enforces demethylation of the locus that encodes PD-1 in antigen-specific CD8(+) T cells. Immunity. 2011;35:400-12.

19. Staron MM, et al. The transcription factor FoxO1 sustains expression of the inhibitory receptor PD-1 and survival of antiviral CD8(+) T cells during chronic infection. Immunity. 2014;41:802-14.

20. Nishimura H, Nose M, Hiai H, Minato N, Honjo T. Development of lupus-like autoimmune diseases by disruption of the PD-1 gene encoding an ITIM motif-carrying immunoreceptor. Immunity. 1999;11:141-51.

21. Nishimura $\mathrm{H}$, et al. Autoimmune dilated cardiomyopathy in PD-1 receptordeficient mice. Science. 2001;291:319-22.

22. Okazaki $T$ et al. Autoantibodies against cardiac troponin I are responsible for dilated cardiomyopathy in PD-1-deficient mice. Nat Med. 2003;9:1477-83.

23. Wang J, et al. Establishment of NOD-Pdcd $1^{-1-}$ mice as an efficient animal model of type I diabetes. Proc Natl Acad Sci U S A. 2005;102:11823-8.

24. Linsley PS, et al. CTLA-4 is a second receptor for the B cell activation antigen B7. J Exp Med. 1991;174:561-9.

25. Walker LS. Treg and CTLA-4: two intertwining pathways to immune tolerance. J Autoimmun. 2013:45:49-57.

26. Iwai Y, Terawaki S, Ikegawa M, Okazaki T, Honjo T. PD-1 inhibits antiviral immunity at the effector phase in the liver. J Exp Med. 2003;198:39-50.

27. Barber $\mathrm{DL}$, et al. Restoring function in exhausted CD8 T cells during chronic viral infection. Nature. 2006:439:682-7.

28. Shiratori T, et al. Tyrosine phosphorylation controls internalization of CTLA-4 by regulating its interaction with clathrin-associated adaptor complex AP-2. Immunity. 1997;6:583-9.

29. Stamper CC, et al. Crystal structure of the B7-1/CTLA-4 complex that inhibits human immune responses. Nature. 2001;410:608-11.

30. Schwartz JC, Zhang X, Fedorov AA, Nathenson SG, Almo SC. Structural basis for co-stimulation by the human CTLA-4/B7-2 complex. Nature. 2001:410:604-8.

31. Qureshi OS, et al. Trans-endocytosis of CD80 and CD86: a molecular basis for the cell-extrinsic function of CTLA-4. Science. 2011:332:600-3.

32. Parry RV, et al. CTLA-4 and PD-1 receptors inhibit T-cell activation by distinct mechanisms. Mol Cell Biol. 2005:25:9543-53.

33. Waterhouse $P$, et al. Lymphoproliferative disorders with early lethality in mice deficient in Ctla-4. Science. 1995:270:985-8

34. Hodi FS, et al. Improved survival with ipilimumab in patients with metastatic melanoma. N Engl J Med. 2010;363:711-23.

35. Topalian SL, et al. Safety, activity, and immune correlates of anti-PD-1 antibody in cancer. N Engl J Med. 2012;366:2443-54.

36. Ishida M, et al. Differential expression of PD-L1 and PD-L2, ligands for an inhibitory receptor PD-1, in the cells of lymphohematopoietic tissues. Immunol Lett. 2002:84:57-62.

37. Yamazaki $T$, et al. Expression of programmed death 1 ligands by murine $T$ cells and APC. J Immunol. 2002:169:5538-45.

38. Eppihimer MJ, et al. Expression and regulation of the PD-LI immunoinhibitory molecule on microvascular endothelial cells. Microcirculation. 2002;9:133-45.

39. Keir ME, et al. Tissue expression of PD-L1 mediates peripheral T cell tolerance. J Exp Med. 2006;203:883-95. 
40. Iwai Y, et al. Involvement of PD-L1 on tumor cells in the escape from host immune system and tumor immunotherapy by PD-L1 blockade. Proc Natl Acad Sci U S A. 2002;99:12293-7.

41. Thompson $\mathrm{RH}$, et al. Costimulatory $\mathrm{B} 7-\mathrm{H} 1$ in renal cell carcinoma patients: Indicator of tumor aggressiveness and potential therapeutic target. Proc Natl Acad Sci U S A. 2004;101:17174-9.

42. Okazaki T, Honjo T. PD-1 and PD-1 ligands: from discovery to clinical application. Int Immunol. 2007;19:813-24.

43. Leach DR, Krummel MF, Allison JP. Enhancement of antitumor immunity by CTLA-4 blockade. Science. 1996;271:1734-6.

44. Peggs KS, Quezada SA, Korman AJ, Allison JP. Principles and use of antiCTLA4 antibody in human cancer immunotherapy. Curr Opin Immunol. 2006;18:206-13.

45. van Elsas A, Hurwitz AA, Allison JP. Combination immunotherapy of B16 melanoma using anti-cytotoxic T lymphocyte-associated antigen 4 (CTLA-4) and granulocyte/macrophage colony-stimulating factor (GM-CSF)-producing vaccines induces rejection of subcutaneous and metastatic tumors accompanied by autoimmune depigmentation. J Exp Med. 1999;190:355-66.

46. Iwai Y, Terawaki S, Honjo T. PD-1 blockade inhibits hematogenous spread of poorly immunogenic tumor cells by enhanced recruitment of effector $T$ cells. Int Immunol. 2005;17:133-44.

47. Nakanishi J, et al. Overexpression of B7-H1 (PD-L1) significantly associates with tumor grade and postoperative prognosis in human urothelial cancers. Cancer Immunol Immunother. 2007:56:1173-82.

48. Ohigashi $Y$, et al. Clinical significance of programmed death-1 ligand-1 and programmed death-1 ligand-2 expression in human esophageal cancer. Clin Cancer Res. 2005;11:2947-53.

49. Nomi T, et al. Clinical significance and therapeutic potential of the programmed death-1 ligand/programmed death-1 pathway in human pancreatic cancer. Clin Cancer Res. 2007;13:2151-7.

50. Hamanishi J, et al. Programmed cell death 1 ligand 1 and tumor-infiltrating CD8+ T lymphocytes are prognostic factors of human ovarian cancer. Proc Natl Acad Sci U S A. 2007;104:3360-5.

51. Wu C, et al. Immunohistochemical localization of programmed death-1 ligand-1 (PD-L1) in gastric carcinoma and its clinical significance. Acta Histochem. 2006;108:19-24.

52. Ghebeh $\mathrm{H}$, et al. The B7-H1 (PD-L1) T lymphocyte-inhibitory molecule is expressed in breast cancer patients with infiltrating ductal carcinoma: correlation with important high-risk prognostic factors. Neoplasia. 2006:8:190-8.

53. Gao Q, et al. Overexpression of PD-L1 significantly associates with tumor aggressiveness and postoperative recurrence in human hepatocellular carcinoma. Clin Cancer Res. 2009;15:971-9.

54. Brahmer JR, et al. Phase I study of single-agent anti-programmed death-1 (MDX1106) in refractory solid tumors: safety, clinical activity, pharmacodynamics, and immunologic correlates. J Clin Oncol. 2010;28:3167-75.

55. Brahmer JR, et al. Safety and activity of anti-PD-L1 antibody in patients with advanced cancer. N Engl J Med. 2012;366:2455-65.

56. Gubin MM, et al. Checkpoint blockade cancer immunotherapy targets tumour-specific mutant antigens. Nature. 2014;515:577-81.

57. Brahmer J, et al. Nivolumab versus Docetaxel in Advanced Squamous-Cell Non-Small-Cell Lung Cancer. N Engl J Med. 2015;373:123-35.

58. Callahan MK, Postow MA, Wolchok JD. Targeting T Cell Co-receptors for Cancer Therapy. Immunity. 2016:44:1069-78.

59. Hamanishi J, et al. Efficacy and safety of anti-PD-1 antibody (Nivolumab: BMS-936558, ONO-4538) in patients with platinum-resistant ovarian cancer. 2014 ASCO Annual Meeting. J Clin Oncol. 2014;32:5s (suppl; abstr 5511).

60. Hamanishi J, et al. Safety and Antitumor Activity of Anti-PD-1 Antibody, Nivolumab, in Patients With Platinum-Resistant Ovarian Cancer. J Clin Oncol. 2015;33:4015-22

61. Hamanishi J, et al. Durable tumor remission in patients with platinumresistant ovarian cancer receiving nivolumab. 2015 ASCO Annual Meeting. J Clin Oncol. 2015;33(suppl; abstr 5570)

62. Hamanishi J, Mandai M, Konishi I. Immune checkpoint inhibition in ovarian cancer. Int Immunol. 2016;28:339-48.

63. Varga A, et al. Antitumor activity and safety of pembrolizumab in patients (pts) with PD-L1 positive advanced ovarian cancer: Interim results from a phase Ib study. 2015 ASCO Annual Meeting. J Clin Oncol. 2015;33(suppl; abstr 5510).

64. Disis ML, et al. Avelumab (MSB0010718C; anti-PD-L1) in patients with recurrent/refractory ovarian cancer from the JAVELIN Solid Tumor phase Ib trial: Safety and clinical activity. 2015 ASCO Annual Meeting. J Clin Oncol. 2015;34(suppl; abstr 5533).
65. Wolchok JD, et al. Nivolumab plus ipilimumab in advanced melanoma. N Engl J Med. 2013;369:122-33.

66. Postow MA, et al. Nivolumab and ipilimumab versus ipilimumab in untreated melanoma. N Engl J Med. 2015:372:2006-17.

67. Hammers $\mathrm{HJ}$, et al. Phase I study of nivolumab in combination with ipilimumab in metastatic renal cell carcinoma (mRCC). 2014 ASCO Annual Meeting. J Clin Oncol.2014;32(suppl; abstr 4504).

68. Patnaik A, et al. Phase 1 study of pembrolizumab (pembro; MK-3475) plus ipilimumab (IPI) as second-line therapy for advanced non-small cell lung cancer (NSCLC): KEYNOTE-021 cohort D. 2015 ASCO Annual Meeting. J Clin Oncol. 2015;33(suppl; abstr 8011).

69. Naidoo J, et al. Toxicities of the anti-PD-1 and anti-PD-L1 immune checkpoint antibodies. Ann Oncol. 2015;26:2375-91.

70. Ahn MJ, et al. 1360: Osimertinib combined with durvalumab in EGFRmutant non-small cell lung cancer: Results from the TATTON phase Ib trial. J Thorac Oncol. 2016;11:S115.

71. Snyder A, et al. Genetic basis for clinical response to CTLA-4 blockade in melanoma. N Engl J Med. 2014;371:2189-99.

72. Rizvi NA, et al. Cancer immunology. Mutational landscape determines sensitivity to PD-1 blockade in non-small cell lung cancer. Science. 2015;348:124-8.

73. Le DT, et al. PD-1 Blockade in Tumors with Mismatch-Repair Deficiency. N Engl J Med. 2015;372:2509-20.

74. Tumeh PC, et al. PD-1 blockade induces responses by inhibiting adaptive immune resistance. Nature. 2014;515:568-71.

75. Hamanishi J, et al. PD-1/PD-L1 blockade in cancer treatment: perspectives and issues. Int J Clin Oncol. 2016:21:462-73.

76. Dudley JC, Lin MT, Le DT, Eshleman JR. Microsatellite Instability as a Biomarker for PD-1 Blockade. Clin Cancer Res. 2016:22:813-20.

77. Ma W, Gilligan BM, Yuan J, Li T. Current status and perspectives in translational biomarker research for PD-1/PD-L1 immune checkpoint blockade therapy. J Hematol Oncol. 2016;9:47.

78. Festino L, et al. Cancer Treatment with Anti-PD-1/PD-L1 Agents: Is PD-L1 Expression a Biomarker for Patient Selection? Drugs. 2016;76:925-45.

79. Muro K, et al. Pembrolizumab for patients with PD-L1-positive advanced gastric cancer (KEYNOTE-012): a multicentre, open-label, phase 1b trial. Lancet Oncol. 2016:17:717-26.

80. Ribas A, et al. Association of response to programmed death receptor 1 (PD-1) blockade with pembrolizumab (MK-3475) with an interferoninflammatory immune gene signature. 2015 ASCO Annual Meeting. J Clin Oncol. 2015;33(suppl; abstr 3001).

81. Mandai M, et al. Anti-PD-L1/PD-1 immune therapies in ovarian cancer: basic mechanism and future clinical application. Int J Clin Oncol. 2016;21:456-61.

82. Sunshine J, Taube JM. Pd-1/Pd-L1 Inhibitors. Curr Opin Pharmacol. 2015;23:32-8

83. Hugo W, et al. Genomic and Transcriptomic Features of Response to AntiPD-1 Therapy in Metastatic Melanoma. Cell. 2016;165:35-44

84. Postow MA. Managing immune checkpoint-blocking antibody side effects. Am Soc Clin Oncol Educ Book. 2015;76-83. doi:10.14694/ EdBook_AM.2015.35.76.

85. Weber JS, Yang JC, Atkins MB, Disis ML. Toxicities of Immunotherapy for the Practitioner. J Clin Oncol. 2015:33:2092-9.

86. Naidoo J, et al. Toxicities of the anti-PD-1 and anti-PD-L1 immune checkpoint antibodies. Ann Oncol. 2016;27:1362

87. Champiat S, et al. Management of immune checkpoint blockade dysimmune toxicities: a collaborative position paper. Ann Oncol. 2016:27:559-74.

88. Ribas A et al. Pembrolizumab versus investigator-choice chemotherapy for ipilimumab-refractory melanoma (KEYNOTE-002): a randomised, controlled, phase 2 trial. Lancet Oncol. 2015;16:908-18.

89. Robert $C$ et al. Pembrolizumab versus Ipilimumab in Advanced Melanoma. N Engl J Med. 2015;372:2521-32

90. Weber JS et al. Nivolumab versus chemotherapy in patients with advanced melanoma who progressed after anti-CTLA-4 treatment (CheckMate 037): a randomised, controlled, open-label, phase 3 trial. Lancet Oncol. 2015;16: 375-84.

91. Garon EB et al. Pembrolizumab for the treatment of non-small-cell lung cancer. N Engl J Med. 2015;372:2018-28.

92. Borghaei $\mathrm{H}$ et al. Nivolumab versus Docetaxel in Advanced Nonsquamous Non-Small-Cell Lung Cancer. N Engl J Med. 2015;373:1627-39.

93. Spira Al et al. Efficacy, safety and predictive biomarker results from a randomized phase II study comparing MPDL3280A vs docetaxel in $2 \mathrm{~L} / 3 \mathrm{~L}$ NSCLC (POPLAR). J Clin Oncol. 2015;33. 
94. Ott PA et al. A phase I study to evaluate the safety and tolerability of MEDI4736, an anti- programmed cell death-ligand-1 (PD-L1) antibody, in combination with tremelimumab in patients with advanced solid tumors. J Clin Oncol. 2015;33.

95. Antonia SJ et al. Phase $1 / /$ l study of nivolumab with or without ipilimumab for treatment of recurrent small cell lung cancer (SCLC): CA209-032. J Clin Oncol. 2015;33.

96. Segal NH et al. Safety and efficacy of MEDI4736, an anti-PD-L1 antibody, in patients from a squamous cell carcinoma of the head and neck (SCCHN) expansion cohort. J Clin Oncol. 2015;33.

97. Seiwert TY et al. Antitumor activity and safety of pembrolizumab in patients (pts) with advanced squamous cell carcinoma of the head and neck (SCCHN): Preliminary results from KEYNOTE-012 expansion cohort. J Clin Oncol. 2015;33.

98. Motzer RJ et al. Nivolumab versus Everolimus in Advanced Renal-Cell Carcinoma. N Engl J Med. 2015;373:1803-13.

99. Rosenberg JE et al. Atezolizumab in patients with locally advanced and metastatic urothelial carcinoma who have progressed following treatment with platinum-based chemotherapy: a single-arm, multicentre, phase 2 trial. Lancet. 2016;387:1909-20.

100. Plimack ER et al. Pembrolizumab (MK-3475) for advanced urothelial cancer: Updated results and biomarker analysis from KEYNOTE-012. J Clin Oncol. 2015;33.

101. Disis ML et al. Avelumab (MSB0010718C; anti-PD-L1) in patients with recurrent/refractory ovarian cancer from the JAVELIN Solid Tumor phase lb trial: Safety and clinical activity. 2016 ASCO Annual Meeting. J Clin Oncol. 2016;34 (suppl; abstr 5533).

102. Ott PA et al. Pembrolizumab in advanced endometrial cancer: Preliminary results from the phase Ib KEYNOTE-028 study. 2016 ASCO Annual Meeting. J Clin Oncol. 2016;34 (suppl; abstr 5581).

103. Frenel et al. Pembrolizumab in patients with advanced cervical squamous cell cancer: Preliminary results from the phase Ib KEYNOTE-028 study. 2016 ASCO Annual Meeting. J Clin Oncol. 2016;34 (suppl; abstr 5515).

104. George et al. Phase 2 study of nivolumab in metastatic leiomyosarcoma of the uterus. 2016 ASCO Annual Meeting. J Clin Oncol. 2016;34 (suppl; abstr 11007).

105. Doi T et al. Updated results for the advanced esophageal carcinoma cohort of the phase Ib KEYNOTE-028 study of pembrolizumab (MK-3475). J Clin Oncol. 2016;34.

106. Fader AN. et al. Preliminary results of a phase II study: PD-1 blockade in mismatch repair-deficient, recurrent or persistent endometrial cancer. The 47th Annual Meeting of the Society of Gynecologic Oncology Late-breaking 2014 (Abstract 3).

107. Sangro B. et al. Safety and antitumor activity of nivolumab (nivo) in patients (pts) with advanced hepatocellular carcinoma (HCC): Interim analysis of dose-expansion cohorts from the phase 1/2 CheckMate-040 study. 2016 ASCO Annual Meeting. J Clin Oncol 34, 2016 (suppl; abstr4078).

108. Emens LA. et al. Inhibition of PD-L1 by MPDL3280A leads to clinical activity in patients with metastatic triple-negative breast cancer. Thirty-Seventh Annual Meeting of CTRC-AACR San Antonio Breast Cancer Symposium, 2015;Abstract P D1-6.

109. Nanda R et al. Pembrolizumab in Patients With Advanced Triple-Negative Breast Cancer: Phase Ib KEYNOTE-012 Study. J Clin Oncol. 2016;34:2460-7.

110. Nghiem PT et al. PD-1 Blockade with Pembrolizumab in Advanced MerkelCell Carcinoma. N Engl J Med. 2016;374:2542-52.

111. Mehnert JM et al. Pembrolizumab for advanced papillary or follicular thyroid cancer: preliminary results from the phase 1b KEYNOTE-028 study. 2016 ASCO Annual Meeting. J Clin Oncol. 2016;34 (suppl; abstr6091).

112. Ansell SM et al. PD-1 blockade with nivolumab in relapsed or refractory Hodgkin's lymphoma. N Engl J Med. 2015;372:311-9.

113. Armand $P$ et al. Programmed Death-1 Blockade With Pembrolizumab in Patients With Classical Hodgkin Lymphoma After Brentuximab Vedotin Failure. J Clin Oncol. 2016.

114. Lesokhin AM et al. Nivolumab in Patients With Relapsed or Refractory Hematologic Malignancy: Preliminary Results of a Phase Ib Study. J Clin Oncol. 2016;34:2698-704.

\section{Submit your next manuscript to BioMed Central and we will help you at every step:}

- We accept pre-submission inquiries

- Our selector tool helps you to find the most relevant journal

- We provide round the clock customer support

- Convenient online submission

- Thorough peer review

- Inclusion in PubMed and all major indexing services

- Maximum visibility for your research

Submit your manuscript at www.biomedcentral.com/submit 